\title{
CICLO BIOLÓGICO DE Rhodnius robustus LARROUSSE, 1927 ALIMENTADO CON SANGRE HUMANA EN CONDICIONES DE LABORATORIO
}

\author{
Elis Aldana, Ezequiel Zamora \& Eliécer Lizano
}

\section{Abstract}

Biological cycle of Rhodnius robustus Larrousse 1927 feeding with human blood in laboratory conditions- The aim of this work was the study of biological cycle of $R$. robustus feeding with human blood. Triatomines were collected in Caño Tigre, state of Mérida, Venezuela. Triatomines were fed through latex membrane with human blood at $37^{\circ} \mathrm{C}$ and sodium citrate as anticoagulant. The results showed 13,9 eggs/ female/week, a 78,32 \% fertility and a biological cycle from egg to adult lasting 70 days. Breeding of $R$. robustus when fed with human blood shows its potential human dwelling domiciliation and Chagas Disease transmission.

Key words: Triatominae, fitness, behavior, Rhodnius robustus

\section{Resumen}

El propósito del presente trabajo es el estudio del ciclo biológico de $R$. robustus alimentado con sangre humana. Los triatominos procedían de

\footnotetext{
Laboratorio de Entomología "Herman Lent", Departamento de Biología, Facultad de Ciencias, Universidad de Los Andes, Núcleo La Hechicera, 5101 Mérida, Venezuela. Fax (58 274 2401286). E-mail: aldana@ula.ve
}

Financiado por el Proyecto C-1199-03-01-B del Consejo de Desarrollo Científico, Humanístico y Tecnológico de la Universidad de Los Andes.

Recebido em : 20/04/2004.

Aceito em: 21/03/2005. 
una colonia fundada con ejemplares capturados en Caño Tigre, estado Mérida, Venezuela. Los insectos fueron alimentados a través de membrana de látex en un alimentador artificial a $37^{\circ} \mathrm{C}$ con sangre humana con citrato de sodio como anticoagulante. Se observó una fecundidad de 13,9 huevos/hembra/semana, fertilidad 78,32\%, el ciclo biológico desde huevo a adulto se completó en aproximadamente 70 días. Los resultados muestran que $R$. robustus es capaz de reproducirse exitosamente al ser alimentada con sangre humana, lo cual contribuye a su potencialidad como especie domiciliaria y transmisora de la Enfermedad de Chagas.

Palabras-clave: Triatominae, eficacia biológica, comportamento, Rhodnius robustus

\section{Introducción}

En Venezuela el vector principal de la Enfermedad de Chagas es Rhodnius prolixus Stål, 1858, aunque Trypanosoma cruzi (Chagas, 1909) se ha encontrado también en condiciones naturales en otros triatomíneos incluyendo al $R$. robustus Larrousse, 1927 (Rossell et al., 1977; Feliciangeli et al., 2002). $R$. robustus es una de las especies de Triatominae que mantiene el ciclo silvestre de $T$. cruzi, es una especie estrechamente relacionada a $R$. prolixus, pero confirmadas como especies distintas por análisis del ADN mitocondrial (Monteiro et al., 2000), morfológico y geométrico (Aldana et al., 2000; Matias et al., 2001), comportamiento de defecación (Aldana et al., 2001) y electroforético de hemoproteínas salivales (Soares et al., 2000). $R$. robustus se encuentra ampliamente distribuida en Venezuela (Tonn et al., 1976), habita en palmas y bromelias y asociada a roedores, marsupiales, murciélagos y aves (Carcavallo et al., 1998a) y se le encuentra en otros países como Bolivia, Brasil, Colombia, Ecuador, Perú y Guyana Francesa (Carcavallo et al., 1998b). Feliciangeli et al. (2002), presentaron evidencias del papel vector extradoméstico de $R$. robustus en el occidente de Venezuela. Los estudios sobre la eficacia reproductiva en triatominos alimentados experimentalmente con sangre humana y otras fuentes animales han sido escasos (Lima Gomes et al., 1990; Guarneri et al., 2000; Aldana et al., 2001; Guarneri et al., 2003). No se conocen parámetros reproductivos de $R$. robustus alimentados con sangre humana; esta información es de 
interés para evaluar la potencialidad de domiciliación de esta especie en la habitación humana. Las primeras observaciones de $R$. robustus en condiciones de laboratorio y alimentando sobre paloma revelaron que esta especie completa su ciclo de huevo a adulto entre 187 a 263 días, el embrión se desarrolla entre 11 a 19 días; los tiempos interestadiales son: I, 14 a 19 días, II, 15 a 40 días, III, 24 a 37 días, IV, 35 a 64 días, V, 51 a 134 días (Jurberg et al., 1970). Alimentando sobre gallina Tonn et al. (1976) encontraron que el ciclo transcurre entre 127 y 224 días, el ciclo huevohuevo entre 149 y 256 días y el desarrollo embrionario entre 12 y 19 días; los tiempos interestadiales son: I, 13 a 19 días, II, 15 a 23 días, III, 22 a 26 días, IV, 25 a 41 días, V, 40 a 96 días. Mascarenha \& Mello (1986) alimentando sobre gallina, realizaron también observaciones sobre el ciclo biológico y parámetros reproductivos con especimenes de $R$. robustus recién capturados en condiciones naturales. Silva \& Silva (1988) alimentando sobre gallina determinaron el efecto de la temperatura sobre el ciclo biológico y parámetros reproductivos de $R$. robustus. Braga et al. (1999) alimentando sobre ratón y paloma, encontraron que el tiempo del ciclo biológico se completa en menor tiempo y la mortalidad de las ninfas es menor, cuando $R$. robustus es alimentado sobre ratón. Rocha et al. (2001), alimentando sobre paloma encontraron que el ciclo biológico de $R$. robustus era afectado por la temperatura y la humedad relativa. EI propósito del presente trabajo es el estudio del ciclo biológico de $R$. robustus alimentando con sangre humana.

\section{Material y Métodos}

Los triatominos procedían de la colonia del Laboratorio de Entomología "Herman Lent" de la Facultad de Ciencias, Universidad de Los Andes, Mérida. La colonia fue fundada en 1973 con ejemplares colectados en Caño de Tigre, estado Mérida, Venezuela. Los insectos fueron mantenidos a 2830 $\mathrm{C}$ y $50 \%$ de humedad relativa. La alimentación con sangre humana a $37^{\circ} \mathrm{C}$ se hizo a través de un alimentador artificial. El alimentador consiste de dos viales de vidrio separados por una membrana de látex. El vial superior incluye un ambiente a temperatura controlada en el cual se coloca la preparación sanguínea a $37^{\circ} \mathrm{C}$. El vial inferior contiene a los triatominos, los cuales alcanzan la membrana de látex colocada en el fondo del vial superior a través de una tira de papel de filtro. 
La sangre procedía de un varón joven y era obtenida por punción venosa con citrato de sodio $0,85 \%$ como anticoagulante. La fecundidad se expresa como el promedio de huevos por hembra por semana. La fertilidad como el porcentaje de huevos eclosionados. La fecundidad se determinó a partir de 49 réplicas de una hembra virgen y de dos machos, de aproximadamente 7-10 días, contados a partir del día de la muda del $V$ estadio a adulto. Los tiempos interestadiales se determinaron a partir de entre $34 \mathrm{y}$ 84 ejemplares de cada estadio, mantenidos los ejemplares de cada estadio en un mismo vial y que hubieran comido hasta repleción con la primera oferta alimenticia. En cada estadio fueron alimentados una muestra diferente de ejemplares y no los mismos del estadio anterior. Una vez alimentados los triatominos, éstos fueron mantenidos a $28^{\circ} \mathrm{C}$ y $50 \%$ de humedad relativa. Esta temperatura fue mantenida en un ambiente estufa de $4 \mathrm{mt}^{2}$ mediante una resistencia eléctrica conectada a un termostato y la humedad fue mantenida con recipientes con agua en volúmenes controlados para mantener dicha humedad. Se mantenía un registro diario de la humedad con un higrómetro portátil de campo. Realizada la primera oferta sanguínea se escogieron aquellos individuos que ad libitum realizaron la ingesta hasta repleción. La segunda y tercera oferta sanguínea se realizaron respectivamente a los 15 y 30 días de la primera.

\section{Resultados y Discusión}

Ciclo biológico de $R$. robustus (Tabla I).

Tiempo de preoviposición: El tiempo de preoviposición fue de 13,87 \pm 5,82 días.

Fecundidad: La fecundidad fue de 13,91 $\pm 3,69$ huevos/hembra/semana. Del total de hembras ensayadas, fueron ovipositoras el $97,9 \%$. En cuanto al tiempo de oviposición, éste fue de 5,91 semanas. Con relación al total de huevos, se obtuvieron 3893 huevos, observación realizada hasta asegurarnos que ninguna hembra ponía más huevos.

Tiempo de desarrollo embrionario: El tiempo transcurrido desde la

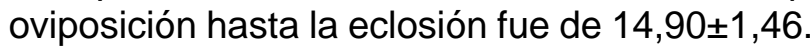

Fertilidad: El porcentaje de huevos eclosionados fue $78,30 \%$.

Tiempos interestadiales: El tiempo transcurrido desde la eclosión del huevo hasta adulto fue aproximadamente de 70 días. De I a II: 11 días; de II a III 11,60 días; de III a IV 11,40 días; de IV a V 13,90 días y de $\mathrm{V}$ a adulto 22,70 días. 
Tabla I

Ciclo biológico y parámetros reproductivos de Rhodnius robustus alimentando con sangre humana.

\begin{tabular}{|c|c|c|c|c|}
\hline $\begin{array}{l}\text { Parámetros } \\
\text { reproductivos }\end{array}$ & $X \pm S D$ & Estadio & $\begin{array}{c}\text { Tiempos } \\
\text { interestadiales } \\
X \pm S D \text { días }\end{array}$ & $\begin{array}{c}\% \\
\text { mortalidad }\end{array}$ \\
\hline $\begin{array}{l}\text { Semanas de } \\
\text { fecundidad }\end{array}$ & & \multirow{4}{*}{$\begin{array}{l}\mathrm{l} \\
\mathrm{n}\end{array}$} & \multirow{4}{*}{$\begin{array}{c}11 \pm 1,6 \\
84\end{array}$} & \multirow{4}{*}{28.2} \\
\hline $\mathrm{n}$ & 48 & & & \\
\hline $\begin{array}{c}\text { Huevos / semana / } \\
\text { hembra }\end{array}$ & $13,9 \pm 3,69$ & & & \\
\hline $\mathrm{n}$ & 48 & & & \\
\hline $\begin{array}{c}\text { Tiempo } \\
\text { preovinosición }\end{array}$ & $13,87 \pm 5,82$ & II & $11,6 \pm 1,3$ & 17.4 \\
\hline $\mathrm{n}$ & 48 & $\mathrm{n}$ & 38 & \\
\hline $\begin{array}{l}\% \text { Hembras } \\
\text { ovipositoras }\end{array}$ & 97.9 & III & $11,4 \pm 1,5$ & 27.6 \\
\hline $\mathrm{n}$ & 49 & $\mathrm{n}$ & 41 & \\
\hline $\begin{array}{c}\text { Huevos totales } \\
n\end{array}$ & $\begin{array}{c}3893 \\
48\end{array}$ & $\begin{array}{l}\text { IV } \\
\mathrm{n}\end{array}$ & $\begin{array}{c}13,9 \pm 3 \\
52\end{array}$ & 7.1 \\
\hline $\begin{array}{l}\text { Tiempo de } \\
\text { desarrollo } \\
\text { embrionario }\end{array}$ & $14,91 \pm 1,46$ & \multirow{3}{*}{$\begin{array}{l}\mathrm{v} \\
\mathrm{n}\end{array}$} & \multirow{3}{*}{$\begin{array}{c}22,7 \pm 5,1 \\
34\end{array}$} & \multirow[t]{3}{*}{28.6} \\
\hline $\mathrm{n}$ & 3049 & & & \\
\hline $\begin{array}{l}\text { Fertilidad total } \% \\
\mathrm{n}\end{array}$ & $\begin{array}{l}78.32 \\
3049\end{array}$ & & & \\
\hline
\end{tabular}

X, media; SD, desviación estándar.

La alimentación con sangre humana reveló que $R$. robustus alcanza valores de fecundidad y fertilidad similares a $R$. prolixus alimentado en condiciones similares (Aldana et al., 2001), pero en $R$. robustus los tiempos interestadiales son más cortos. Si se comparan los resultados del presente trabajo con los de Jurberg et al. (1970) y Rocha et al. (2001) alimentando sobre paloma y Tonn et al. (1976) alimentando sobre gallina, encontramos que en $R$. robustus alimentando con sangre humana la mortalidad de ninfas es más alta y el ciclo biológico es más corto.

El periodo del ciclo biológico, la fecundidad promedio por hembra y el período de fecundidad alimentando sobre gallina reportados por Mascarenha \& Mello (1986), fueron similares a los correspondientes valores obtenidos en nuestro trabajo aunque las desviaciones estándares de 
la fecundidad promedio por hembra y el período de fecundidad fueron notablemente mayores. Esta diferencia en las desviaciones estándares se debe quizá a que nuestros resultados fueron obtenidos a partir de una colonia mantenida durante 30 años en condiciones estables de humedad, temperatura y alimentación, mientras que Mascarenha \& Mello (1986) obtuvieron sus resultados a partir de especimenes recién colonizados y procedentes de condiciones naturales. Las condiciones estables de la colonia de nuestro laboratorio posiblemente han seleccionado unas variaciones genotípicas de los especimenes menos amplias, mientras que en condiciones naturales las exigencias del medio son variables, seleccionando así una mayor variedad genotípica. Por otra parte, la fertilidad observada por nosotros duplica a la observada por Mascarenha \& Mello (1986). Cabe destacar sin embargo que la fertilidad reportada por estos autores es dudosa pues según ellos fue $40 \pm 41,3$, es decir, la desviación estándar supera la media.

Silva \& Silva (1988) alimentando sobre gallina estudiaron la influencia de la temperatura $\left(25^{\circ} \mathrm{C}\right.$ y $\left.30^{\circ} \mathrm{C}\right)$ sobre algunos parámetros reproductivos y el periodo del ciclo biológico de $R$. robustus procedentes de una colonia en condiciones de laboratorio. Estos autores encontraron que estas dos temperaturas afectan distintamente los parámetros estudiados. Nuestras observaciones fueron realizadas manteniendo a los insectos a $28-30^{\circ} \mathrm{C}$, sin embargo los valores obtenidos fueron notablemente menores a los correspondientes valores obtenidos por Silva \& Silva (1988). Dado que en ambos casos se trata de colonias procedentes de condiciones de laboratorio, las diferencias observadas pueden deberse a las distintas fuentes alimenticias, sangre humana en nuestro caso y gallina en la de los autores mencionados.

Se concluye por tanto que $R$. robustus presenta eficacia reproductiva alimentando con sangre humana, dado los valores de fecundidad, fertilidad y el tiempo más corto del ciclo biológico, pero es más afectada la tasa de mortalidad de las ninfas.

\section{Referencias Bibliográficas}

Aldana, E.; Lizano, E.; Contreras, F.; Valderrama, A. \& Viera, D., 2000. Estudio morfológico de estadios ninfales de varias especies del género Rhodnius (Hemiptera: Reduviidae). Caldasia 22:347-351. 
Aldana, E.; Lizano, E. \& Valderrama, A., 2001. Efecto de la alimentación con sangre humana sobre la fecundidad, fertilidad y ciclo biológico de Rhodnius prolixus (Hemiptera: Reduviidae). Rev. Biol. Trop. 49:689-692.

Braga, M.V.; Prata, K.C. \& Brazil, R.P., 1999. Biology of nymphs of Rhodnius robustus Larrousse, 1927 (Hemiptera, Reduviidae), fed on pigeon or on swiss mouse blood in laboratory conditions. Rev. Bras. Biol. 59:91-94.

Carcavallo, R.; Rodríguez, M.E.F. \& Salvatella, R. et al., 1998a. Habitats and related fauna. Volumen 2:561-600. In: Atlas dos Vetores da Doença de Chagas nas Américas (R.U. Carcavallo, I. Galíndez Girón, J. Jurberg \& H. Lent eds.), Editora Fiocruz, Rio de Janeiro. 302 p.

Carcavallo, R.; Curto de Casas, S.I. \& Sherlock, I.S. et al., 1998b. Geographical distribution and alti-latitudinal dispersion of Triatominae. Volumen 3:747-792. In: Atlas dos Vetores da Doença de Chagas nas Américas (R.U. Carcavallo, I. Galíndez Girón, J. Jurberg \& H. Lent eds.), Editora Fiocruz, Rio de Janeiro. 471 p.

Feliciangeli, D.; Dujardín, J. \& Bastrenta, B. et al, 2002. Is Rhodnius robustus (Hemiptera:Reduviidae) responsible for Chagas disease tranmission in Western Venezuela? Trop. Med. Int. Health 7:280-287.

Guarneri, A.A.; Pereira, M.H. \& Diotaiuti, L., 2000. Influence of the blood meal source on the development of Triatoma infestans, Triatoma brasiliensis, Triatoma sordida, and Triatoma pseudomaculata (Heteroptera, Reduviidae). J. Med. Entomol. 37:373-379.

Guarneri, A.A.; Diotaiuti, L.; Gontijo, N.F.; Gontijo, A.F. \& Pereira, M.H., 2003. Blood-feeding performance of nymphs and adults of Triatoma brasiliensis on human hosts. Acta Trop. 86: 361-370.

Jurberg, J.; Reis, V. \& Lent, H., 1970. Observações Sôbre o Ciclo Evolutivo, em Laboratório, do Rhodnius robustus Larrousse, 1927 (Hemiptera, Reduviidae, Triatominae Rev. Bras. Biol. 30:477-481.

Lima, Gomes J.; Azambuja, P. \& Garcia, E., 1990. Comparative studies on the growth and reproductive performances of $R$ hodnius prolixus reared of different blood sources. Mem. Inst. Oswaldo Cruz 85:299-304. 
Matias, A.; de la Riva, J.X.; Torrez, M. \& Dujardin, J.P., 2001. Rhodnius robustus in Bolivia identified by its wings. Mem. Inst. Oswaldo Cruz 96:947-950.

Mascareñas, B.M. \& Mello, J.A.S.N. de, 1986/87. Triatomíneos da Amazônia: ocurrência de triatomíneos na área do reservatório da Hidreléctrica de Tucuruí (Pará) e Observaçôes sobre o ciclo evolutivo de Rhodnius robustus Larrousse, 1927 (Hemiptera, Reduviidae, Triatominae). Acta Amazonica 16/17:607-616.

Monteiro, F.A.; Wesson, D.M.; Dorson, E.M.; Schofield, C.J. \& Beard, C.B., 2000. Phylogeny and molecular taxonomy of the Rhodniini derived from mitochonrial and nuclear DNA sequences. Am. J. Trop. Med. Hyg. 62:460-465.

Rocha, D.S.; Jurberg, J.; Carvallo, R.; Presgrave, O.; Cunha, V. \& Galvão, C., 2001. Influência da temperatura a umidade no desenvolvimento ninfal de Rhodnius robustus. Rev. Saude Publica. 35:400-406.

Rossell, O.; Mogollón, J. \& Pacheco, J., 1977. Presencia de Rhodnius robustus Larrousse, 1927 (Hemiptera, Reduviidae) en el estado Trujillo, Venezuela (Comunicación preliminar). Bol. Dir. Malariol. Saneam. Amb. 17:230-233.

Silva, I.G. da \& Silva, H.H. da, 1988. Influencia da temperatura na biologia de Triatomíneos. XI. Rhodnius robustus Larrousse 1927 (Hemiptera, Reduviidae). Rev. Goiana Med. 34:145-154.

Soares, R.P.; Sant'Anna, N.F.; Gontijo, N.F.; Romanha, A.J.; Diotaiuti, L. \& Pereira, M.H., 2000. Identification off morphologically similar Rhodnius species (Hemiptera: Reduviidae: Triatominae) by electrophoresis of salivary heme proteins. Am. J. Trop. Med. Hyg. 62: 157-161.

Tonn, R.J.; Carcavallo, R. \& Ortega, R., 1976. Notas sobre la biología, ecología y distribución geográfica de Rhodnius robustus Larrouse, 1927 (Hemiptera, Reduviidae). Bol. Dir. Malariol. Saneam. Amb. 16:158-162. 